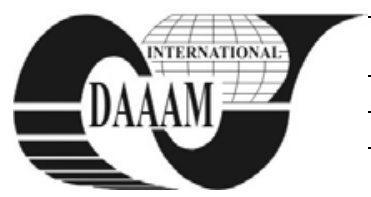

Annals of DAAAM for 2011 \& Proceedings of the 22nd International DAAAM Symposium, Volume 22, No. 1, ISSN 1726-9679 ISBN 978-3-901509-83-4, Editor B. Katalinic, Published by DAAAM International, Vienna, Austria, EU, 2011 Make Harmony between Technology and Nature, and Your Mind will Fly Free as a Bird Annals \& Proceedings of DAAAM International 2011

\title{
MODAL ANALYSIS OF THE METALLIC MEMBRANE FROM ELASTIC COUPLING STRUCTURE
}

\author{
DOBRE, D[aniel]; SIMION, I[onel]; IONITA, E[lena] \& DOBRESCU, T[iberiu] G[abriel]
}

\begin{abstract}
The paper describes the modal analysis used to extract the natural frequencies and mode shapes of a flexible membrane from elastic coupling structure. A membrane should be designed to produce natural frequencies that will prevent the coupling component from vibrating at one of its fundamental modes under operating conditions. The modal analysis is developed on paper for one membrane. This study and the corresponding results are given and discussed. Modal analysis is important as a precursor to any dynamic analysis because knowledge of the membrane's fundamental modes and frequencies can help characterize its dynamic response and decrease the vibrations' amplitudes.
\end{abstract}

Key words: flexible coupling, modal analysis, mode shape vibration, natural frequency

\section{INTRODUCTION}

Reliability and efficiency are main problems that support high power transmissions renovations. Elastic couplings are, as usual, industrial assemblies used to transmit rotating movement torque and compensate angular and radial misalignments of the connected end shafts. Flexible couplings connect two rotating shafts, but are designed to dampen vibration, absorb some of the shock loading and provide some axial movement or end float of the shafts, as well as compensate for minor misalignment. Transferring the torque transmission, the rotating coupling vibrates mainly by the dynamic forces acting from unbalance, misalignment and from coupling elements motion. The coupling's dynamics depends on the membrane's packet motion and relative motion between each semi-coupling. The high rotating velocity generates large inertia forces acting on membranes units (Mancuso, 1986).

The flexible membranes have a spoked form, the spokes' deformation giving the coupling its flexibility and ability to handle installation misalignments. It is necessary and adequate to perform some complexe researches on flexible intermediary membranes from elastic coupling structure, which would lead to working optimization on a long period, with favorable results in what concerns life and safeness of the system (Dobre, 2004).

Flexible element couplings have an axial natural frequency. The coupling will vibrate at its axial natural frequency only if it is excited by the connected machines.

This paper is focused on the issue of calculating the frequencies and mode shape vibrations of a metallic membrane by the Finite Element Analysis.

\section{THEORETICAL CONSIDERATIONS}

Modal analysis is used to extract natural frequencies and mode shapes of a flexible membrane. Modal analysis is important as a precursor to any dynamic analysis because knowledge of the membrane's fundamental modes and frequencies help characterize its dynamic response.

Modal analysis in the ANSYS program is based on the following equation of motion for a finite element system:

$$
[M]\{\ddot{u}\}+[C]\{\dot{u}\}+[K]\{u\}=0
$$

where:

- $[\mathrm{M}]$ is the mass matrix,

- [C] is the damping matrix,

- $[\mathrm{K}]$ is the stiffness matrix,

- $\{\ddot{u}\}$ is the nodal acceleration vector,

- $\{\dot{u}\}$ is the nodal velocity vector,

- $\{u\}$ is the nodal displacement vector.

In the undamped case (which is most common for modal analysis) the damping term, $[\mathrm{C}]\{\dot{u}\}$, is ignored and the equation reduces to:

$$
\left([K]-\omega^{2}[M]\right)\{\bar{u}\}=0
$$

where $\omega^{2}$ (the square of natural frequencies) represents the eigenvalues and $\{\bar{u}\}$ (the mode shapes which do not change in time) represents the eigenvectors.

The roots of equation (2) are $\omega_{i}^{2}$, the eigenvalues, where $i$ ranges from 1 to number of DOF. The square roots of the eigenvalues are $\omega_{i}$, the structure's natural circular frequencies (radians/sec). Natural frequencies $f_{i}$ are then calculated as:

$$
f_{i}=\frac{\omega_{i}}{2 \pi}\left(\frac{\text { cycles }}{\sec }\right)
$$

The eigenvectors $\{u\}_{i}$ represent the mode shapes - the shape assumed by the structure when vibrating at frequency $f_{i}$.

\section{MODAL ANALYSIS OF THE MEMBRANE}

The modal analysis is the main objective of the paper. For modal analysis with FEM there were used SHELL type elements with 4 nodes (QUAD4), ANSYS 6.1 package being very helpful. This analysis was made for the following parameters: torque of $50 \mathrm{~N} \cdot \mathrm{m}$, rotation speed of $4500 \mathrm{rpm}$, thickness $\mathrm{h}=0.3 \mathrm{~mm}$ (the membrane depth is very small), modulus of elasticity $E=2.06 \cdot 10^{5} \mathrm{~N} / \mathrm{mm}^{2}$, density $\rho=7800$ $\mathrm{kg} / \mathrm{m}^{3}$, Poisson's coefficient $v=0.33$ and shear modulus $\mathrm{G}=8 \cdot 10^{4} \mathrm{~N} / \mathrm{mm}^{2}$. The values of these parameters were taken from specialized literature in this field.

Several observations are made in connection with the load scheme and boundary conditions for FEA:

- the pressure distribution in the outer holes for assembling with the counterpart piece (hub) is considered uniform on semi-cylindrical surface in the sense of the driving torque.

- the inner assembling holes are blocked as rotation and translation on contour nodes (boundary conditions on the inner holes emphasize the avoidance degrees of freedom of rotation and translation movements at selected nodes).

For the modal analysis of the membrane, the following situations were analyzed:

a) all outer holes are free as displacement and rotation on the direction of the membrane axis;

b) all outer holes are blocked as displacement on the direction of the membrane axis. 


\section{RESULTS. DISCUSSION}

No inertial discontinuities have been considered. The 10 proper frequencies and the corresponding modes shape vibrations have been determined. The proper frequencies for these two cases of modal analysis are indicated in table 1 .

\begin{tabular}{|c|c|c|}
\hline $\begin{array}{c}\text { Vibration } \\
\text { mode }\end{array}$ & $\begin{array}{c}\text { Without blockages } \\
\text { on the outer holes }\end{array}$ & $\begin{array}{c}\text { With blockages on } \\
\text { the outer holes }\end{array}$ \\
\hline 1 & 157,5 & 2067 \\
\hline 2 & 157,5 & 2087 \\
\hline 3 & 157,6 & 2087 \\
\hline 4 & 198 & 2139 \\
\hline 5 & 198 & 2139 \\
\hline 6 & 308 & 2176 \\
\hline 7 & 373 & 2534.5 \\
\hline 8 & 575 & 2541 \\
\hline 9 & 575 & 2541 \\
\hline 10 & 879 & 2556 \\
\hline
\end{tabular}

Tab. 1. Results obtained by modal analysis

The natural frequencies for 10 vibration modes have values in the interval $f \in[2067,2556]$. This means that the membrane is stable at vibrations.

The mode shapes are presented in a space representation in gray shades (the original figures have the mode shapes defined by different colours). Due to the limited space of the paper only three modes shape vibrations have been given (fig. $1 \div 3$ ).
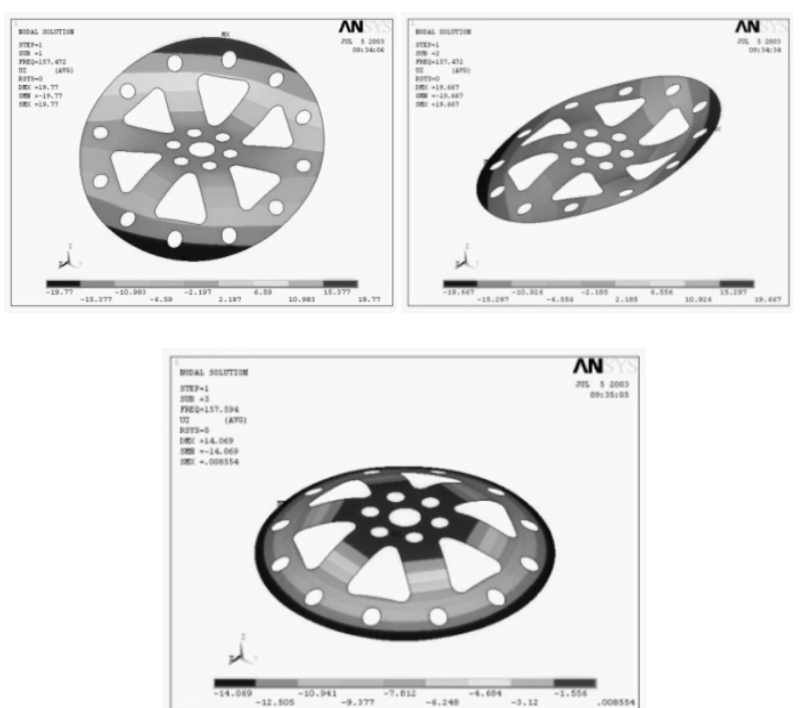

Fig. 1. First three modes shape vibrations without blockages on the outer holes

The input parameters of a membrane that are subject to change are geometric parameters such as fillet radius or spokes' width but may be also loads locations or constraints location, thereby reducing dynamic loading and extending coupling life (Mancuso, 1986).

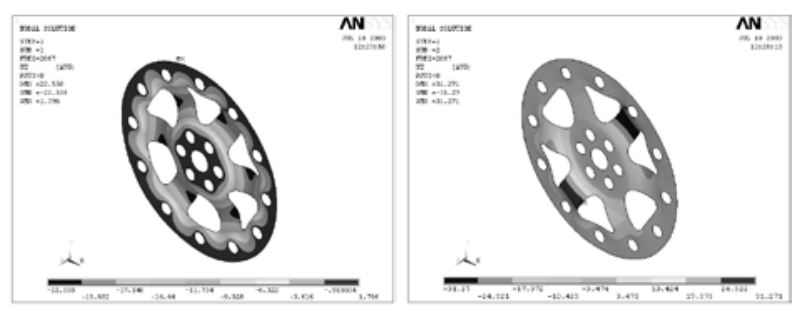

Fig. 2. First and second modes shape vibrations with blockages on the outer holes

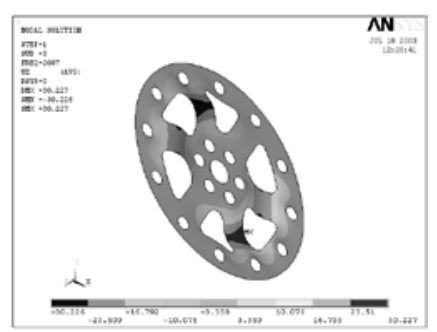

Fig. 3. Third mode shape vibration with blockages on the outer holes

The variation curve of proper frequency versus vibration mode is illustrated in figure 4 .

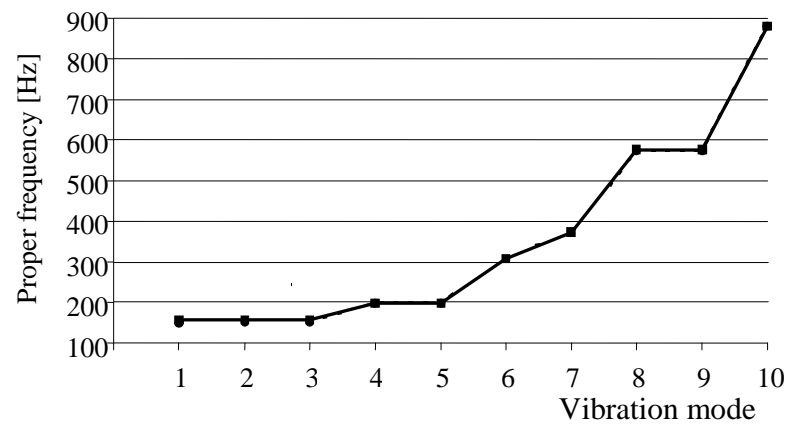

Fig. 4. Proper frequencies versus vibration modes

Due to symmetry, the first and second modes have similar shapes, which were obtained at approximately the same frequency, vibrations taking place around two perpendicular axes. The torque loading changes insignificantly their elastic membrane vibration frequencies (Sorohan \& Sandu, 1997).

The results of the evaluation are used to increase efficiency and feasibility of the membrane design.

\section{CONCLUSION}

Modal analysis is useful for studying the effects of loading conditions and boundary constraints. A metallic membrane should be designed to produce natural frequencies that will prevent the coupling component from vibrating at one of its fundamental modes under operating conditions.

A FEA model was necessary for calculations, in order to determine the vibration modes and axial natural frequencies. Excitation of the membrane at near natural frequency could be responsible for the vibration problem in elastic coupling structure. Also, knowing the mode shapes' vibration can determine the structure's reinforcing measures in order to reduce vibration amplitudes.

It is relevant to state that an increase of dynamic torsional stiffness causes an increase in the resonant speeds. This is important because the resonant speeds should be present above the operating speed range (Birsan \& Jascanu, 1998).

The results are important for other researches in the case of entire membrane packet.

\section{REFERENCES}

Birsan, J.G. \& Jascanu, M. (1998). Elastic couplings’ dynamic, Tehnica Publishing House, ISBN 973-31-1232-1, Bucharest Dobre, D. (2004). Researches on multi-criteria optimization of elastic couplings with metallic flexible membranes, $\mathrm{PhD}$ Thesis, University Politehnica of Bucharest

Mancuso, J. (1986). Disc vs. Diaphragm Couplings, Machine Design, Vol. 58, No. 17, 24 July 1986, pp. 95-98

Sorohan, S. \& Sandu M.A. (1997). Nonlinear FEM-Analysis of a Diaphragm Spring, ELFIN 4, pp. 125-128, Constanta, Romania 